\title{
Ayuda entre Iguales en las escuelas: desarrollo y retos
}

\author{
Helen Cowie ${ }^{1}$ \\ $\mathbf{y}$ \\ F. Javier Fernández ${ }^{2}$
}
${ }^{1}$ European Institute of Health and Medical Sciences
University of Surrey, Guildford
${ }^{2}$ Departmento de Psicología Evolutiva y Educación
Universidad de Málaga, Málaga

Reino Unido y España

h.cowie@surrey.ac.uk 


\section{Resumen}

En este trabajo describimos los programas de Ayuda entre Iguales, es decir, diferentes estrategias llevadas a cabo por los jóvenes para combatir el problema de la violencia en sus escuelas. Los métodos que detallamos están diseñados para ayudar a crear contextos escolares donde las relaciones personales son valoradas, y donde el respeto mutuo y la cooperación son la forma con que los alumnos de estas escuelas se relacionan unos con otros. Primero, comentamos la naturaleza de la ayuda entre iguales y algunas de las formas en que se ha desarrollado a lo largo del tiempo. Segundo, describimos algunas investigaciones y analizamos sus resultados. Y finalmente, esbozamos algunas conclusiones sobre el papel desarrollado por los programas de ayuda entre iguales en las escuelas y sus implicaciones en un contexto social más amplio.

Palabras claves: Ayuda entre iguales, bullying, violencia escolar, resolución de conflictos, participación activa de los niños. 


\section{Introducción}

Podríamos argumentar que la causa endémica de los problemas de la sociedad moderna es el abuso de poder, particularmente cuando los que practican ese abuso no son conscientes de ello. La escuela, como un microsistema de la sociedad, crea un contexto donde los niños experimentan poder, y algunos llegan a familiarizarse con la opresión que unos pocos realizan. Los oprimidos acaban aceptando como natural la posición privilegiada de algunos iguales y construyen una imagen de sí mismos como subordinados, e internalizan un sentimiento de baja autoestima y un elevado resentimiento. A su vez, los dominadores pueden también sufrir daños emocionales. Estos no pueden correr el riesgo de ser conscientes de sus propios sentimientos y emociones, ya que podrían llegar a ser sensibles al dolor de aquellos de los que abusa. Las escuelas pueden, y deberían, jugar un papel importante en el desafío del maltrato entre iguales, creando una atmósfera de cuidado, es decir, una atmósfera donde los alumnos son animados a ayudar a otros, y con claras sanciones contra el bullying. Para ello, los profesores pueden promover valores de cooperación y solidaridad en sus clases. Pero esto puede no ser suficiente, ya que en el patio de recreo los niños hacen frente a fuertes presiones de los iguales para inhibirse a la hora de ofrecer ayuda a un compañero en una situación de malestar, ya sea por miedo a represalias, por falta de seguridad en si mismo, por miedo a ser rechazado, o simplemente por dudas sobre no saber decir o hacer lo adecuado en esa situación.

La investigación llevada a cabo por Salmivalli, Lagerspetz, Björkqvist, Österman y Kaukiainen (1996) en Finlandia, ha posibilitado una mayor comprensión del papel de los espectadores o testigos del bullying a la hora de actuar, o no, para contrarrestar la violencia entre compañeros. Salmivalli y colegas encontraron que es posible asignarle un papel como partícipes al $87 \%$ de los estudiantes presentes en un episodio de bullying. Además de los agresores y las victimas, otras figuras identificadas son los asistentes, aquellos que ayudan físicamente al agresor; los reforzadores, aquellos que incitan y fomentan la violencia y la exclusión social; los observadores, aquellos que se mantienen neutrales e inactivos y pretenden no ver lo que está sucediendo; y muy pocos son defensores, aquellos que aportan ayuda a las víctimas y hacen frente al agresor. La mayoría de los espectadores desaprueban el bullying y a una proporción le gustaría ayudar a los compañeros de forma voluntaria, pero a 
menudo se encuentran inseguros sobre que deberían hacer para intervenir, o están demasiados confusos o avergonzados por la situación como para ser capaces de ofrecer un apoyo útil (Shulman, 2002). Batson, Ahmad, Lishner y Tsang (2002), identificaron la base de este comportamiento altruista como una "emoción empática hacia una persona necesitada". Según estos investigadores, los programas de socialización deberían fomentar la toma de perspectiva y la empatía como medios para aumentar la capacidad de los individuos de desarrollar relaciones más satisfactorias, así como una mayor tolerancia hacia los grupos estigmatizados.

En la prevención de los episodios de maltrato, los iguales juegan un papel importante en la protección de sus amigos, actuando en algunos casos en su lugar para evitar su victimización, así como, haciendo menos probable que sean objeto de ataques futuros (Boulton, Trueman, Chau, Whitehand y Amatya, 1999). Una adecuada manera de extender esta protección mas allá del más íntimo círculo de amistad es creando contextos donde exista optimismo y esperanza en que las cosas y las relaciones pueden ir mejor (Seligman, 1995). Así pues, las escuelas pueden contribuir a la mejora de las relaciones interpersonales fomentando entre sus alumnos valores morales basados en los principios de igualdad, preocupación, tolerancia y empatía por los sentimientos de los demás (Schulman, 2002).

Un método que puede jugar un importante papel en el desarrollo de estos valores y en el desafío de la violencia escolar es la Ayuda entre Iguales (Peer Support). En las escuelas y contextos donde los métodos de ayuda entre iguales están en acción, es más probable que las actitudes de los compañeros, en general, puedan cambiar hacia una postura de mayor apoyo hacia las víctimas. Alrededor del 30\% de los escolares que son víctimas de maltrato de iguales lo sufren en silencio (Naylor y Cowie, 1999). En este contexto los espectadores pueden jugar un papel crucial a la hora de defender a los iguales en riesgo de victimización. Como muchas escuelas están descubriendo, la ayuda entre iguales puede ser una forma dinámica y flexible de combatir el problema de la violencia escolar. A través de ella, los defensores y los observadores a menudo formarán redes de apoyo y alargarán la mano a las victimas, que de lo contrario serían excluidas o rechazadas por el grupo de iguales.

\section{¿Qué es la ayuda entre iguales?}

Los sistemas de ayuda entre iguales aportan a los jóvenes un marco desde el cual pueden desarrollar habilidades que le permitan desafiar las agresiones cuando se produzcan, 
obteniendo a su vez experiencia en la promoción de valores de no violencia en el contexto escolar. Estos sistema no involucran a los protagonistas directos de una situación de bullying, pero sí a los testigos, especialmente a esos que observan el bullying y les gustaría jugar un papel activo en su prevención.

Cowie y Wallace (2000) identificaron las principales características de la ayuda entre iguales:

- Los jóvenes son entrenados para trabajar juntos fuera de su círculo de amistad. Este tipo de interacción ayuda a reducir los prejuicios y fomenta la confianza entre género y grupos étnicos.

- Se les da a los jóvenes la oportunidad, a través de un adecuado entrenamiento, de aprender habilidades de comunicación, de compartir información y de reflexionar sobre sus propias emociones.

- Los jóvenes son entrenados para tratar con conflictos y ayudar a los compañeros a relacionarse los unos con los otros de una forma más constructiva y no violenta.

Algunas de las claves de la efectividad de los sistemas de ayuda entre iguales, identificadas por Cowie y Wallace (2000), son las siguientes:

- Los iguales son capaces de detectar la violencia en etapas más tempranas que los profesores y otros adultos.

- Es mas probable que los jóvenes confíen en algún compañero que en un adulto.

- Las víctimas de la violencia tienen a alguien a quien acudir, y perciben a la escuela actuando sobre el problema.

- A los profesores a menudo les falta tiempo y recursos para ocuparse de todos los problemas interpersonales que les llegan a lo largo de un día.

- Los ayudantes consiguen valiosas habilidades interpersonales y disponen de un marco dentro del cual pueden aprender sobre ciudadanía activa.

- Con el tiempo, la escuela es percibida por los padres como una organización que cuida y se preocupa por el bienestar de sus estudiantes. 
- Los sistemas de ayuda entre iguales ofrecen importantes relaciones potenciales con otros servicios de la escuela, como por ejemplo, el departamento de orientación.

- Entre los ayudantes a menudo se encuentra un importante número de jóvenes que con anterioridad han sido víctimas de violencia. Esto les hace tener empatía por la experiencia, además de, permitir su integración en un grupo de iguales en el que predomina el apoyo y la ayuda.

Tipos de ayuda entre iguales

Es importante distinguir entre los diferentes sistemas de ayuda entre iguales que están disponibles, y seleccionar el más adecuado para las necesidades de la escuela. Los principales tipos de ayuda entre iguales, llevados a cabo exitosamente en escuelas europeas, son los siguientes:

- La hora del círculo (Circle Time). Un maestro lidera y dinamiza a un grupo de alumnos para que estos compartan sus sentimientos y preocupaciones, y si es posible, busquen diferentes alternativas para mejorar la situación. De esta forma los alumnos se sienten apoyados y trabajan conjuntamente en la mejora de las relaciones interpersonales. Su frecuencia suele ser semanal.

- Compañeros amigos (Befriending). Son voluntarios mayores o de la misma edad de los compañeros a los que ayudan, entrenados en habilidades interpersonales, tales como escucha activa, asertividad y liderazgo, para ofrecer apoyo y amistad de forma informal a aquellos que son más vulnerables debido a la soledad, exclusión social o victimización. Este apoyo se produce, por ejemplo, jugando o hablando en el recreo con los compañeros en riesgo de victimización.

- Mediación y resolución de conflictos (Mediation and conflict resolution). Se trata de un proceso estructurado en el cual los alumnos voluntarios son entrenados para ayudar a resolver los conflictos entre dos compañeros. Los mediadores ayudan a sus compañeros a encontrar una solución satisfactoria para ambas partes. 
- Escucha activa (Active Listening). En este sistema los alumnos ayudantes son entrenados, normalmente por un psicólogo, en habilidades de escucha activa para poder apoyar a algún compañero en situación de malestar. La supervisión por parte del psicólogo o de los profesores encargados de este sistema es una parte esencial del programa. La ayuda es prestada de manera más formal y estructura, y los encuentros se suelen dar en una habitación de la escuela destinada a tal uso.

- Tutorización de iguales (Mentoring). En este método los alumnos más mayores son entrenados para ofrecer guía y modelos de conducta a otros alumnos más jóvenes a través de un contacto individual. El tiempo que comparten estos alumnos es empleado en juegos estructurados, ayuda con los deberes, y la posibilidad de hablar abiertamente sobre sentimientos y preocupaciones. Los alumnos tutores actúan como modelos, potenciando la colaboración y ofreciendo consejos apropiados.

Los programas de Ayuda entre Iguales varían ampliamente según las escuelas, y los grupos de edad en que son implantados, produciéndose ajustes en la selección de los sistemas mas adecuados (ver tabla 1). En las escuelas de primaria los proyectos generalmente van dirigidos a la identificación y apoyo de los alumnos que se encuentran a menudo solos en el patio de recreo, y predomina el entrenamiento de los alumnos ayudantes en las formulas de compañeros amigos o compañeros de juego. En las escuelas de educación secundaria los proyectos a menudo suponen, para los ayudantes de iguales, trabajar en métodos de resolución de conflictos interpersonales, de tutorización de un grupo de alumnos mas jóvenes, o en contactos individuales con alumnos con dificultades de adaptación (Cowie y Wallace, 2000; Smith y Watson, 2004).

Tabla 1. Grupos de edades a los que pueden ser más efectivos los diferentes sistemas de ayuda entre iguales
Tipo de sistema
7-9 anos
9-11 anos
11-18+ anos

\begin{tabular}{cccc}
\hline La hora del circulo & SI & SI & SI \\
Compañeros amigos & SI & SI & SI \\
Mediación/Resolución de & NO & SI & SI \\
conflictos & NO & NO & SI \\
Escucha activa & SI & SI & SI \\
Tutorización de iguales & & & \\
\hline
\end{tabular}




\section{Ayuda entre iguales e Internet}

Los proyectos de Ayuda entre Iguales evolucionan y sufren pequeñas modificaciones a lo largo del tiempo en que son implementados, en función de las necesidades locales y la percepción de los alumnos sobre la efectividad y aceptación de este tipo de intervenciones. Con el avance de la tecnología, los métodos de ayuda entre iguales también tienen en cuenta las posibilidades de ayuda a distancia, como puede ser el uso de Internet y del correo electrónico (Cartwright, 2005; Cowie y Hutson, 2005). Un estudio realizado en los Estados Unidos halló que el $97 \%$ de los jóvenes entre 12 y 18 años usaban Internet (Ybarra, 2004). Internet es un lugar dinámico para la gente joven, el cuál puede facilitar la comunicación social (Crecer y Balme, 2001), y proporcionar información adecuada sobre las cuestiones que afectan a los jóvenes, por ejemplo a través de páginas web sobre bullying (Borzekowski y Rickert, 2001).

Además, para aquellos jóvenes que se sienten carentes de relaciones familiares y de iguales saludables, las relaciones en línea pueden resultar un sustitutivo (Wolak, Kimberly y Finkelhor, 2003). Internet ha sido anunciado como un lugar donde se permite derribar las barreras, favoreciendo de esta forma el contacto entre personas que podrían, de otra forma, llegar a ser marginadas (McKenna y Bargh, 2000). A través de Internet los grupos socialmente rechazados son capaces de encontrar el apoyo social del que a menudo carecen en sus relaciones tradicionales (Ybarra y Mitchell, 2004).

En el año 2000 una escuela en el norte de Inglaterra introdujo un programa de ayuda entre iguales, BULLYinc, para fomentar las relaciones entre los estudiantes más jóvenes y los mayores de la escuela. Un grupo voluntario de ayudantes fueron entrenados en terapia de reevaluación. El sistema de ayuda entre iguales preferido en esta escuela fue el contacto a través del correo electrónico. Para proporcionar ayuda individualizada a los alumnos con problemas se creó una dirección de correo electrónico y se colocó un buzón de cartas en el vestíbulo de la escuela, siendo ambos medios revisados regularmente por los ayudantes. La ayuda a los compañeros necesitados era proporcionada únicamente a través del correo electrónico, o bien, se persuadía al emisor del mensaje para que asistiera a una reunión informal a la hora del almuerzo. 
En general, los programas de ayuda entre iguales han producido influencias positivas en las escuelas, desarrollando en ellas una mayor educación emocional y potenciando la reflexión de los alumnos sobre el impacto de sus acciones en los demás (Cartwright, 2005). A continuación, veremos detalladamente los resultados de algunas investigaciones que han analizado la efectividad y los beneficios de estos programas.

\section{¿Qué nos dicen las investigaciones?}

Los programas de Ayuda entre Iguales han crecido en popularidad en los últimos años y en la actualidad son ampliamente usados como intervención para combatir la violencia y la exclusión social, así como, para promover bienestar y ajuste emocional entre los estudiantes, tanto en educación primaria como secundaria (Cowie, Boardman, Dawkins y Jennifer, 2004).

A la hora de analizar los resultados de los programas de Ayuda entre Iguales nos encontramos ante la dificultad de la heterogeneidad de las evaluaciones realizadas. Son muchos los estudios basados en impresiones personales de estudiantes y profesores, recogidas por profesionales entusiastas que introdujeron y llevaron a cabo la intervención. Sin embargo, otras han seguido una evaluación independiente, pero varían en la naturaleza de los datos recogidos, desde resúmenes generalizados apoyados por citas de apoyo a la intervención, hasta informes cuantitativos de actitudes y resultados en diferentes áreas. Estos estudios han apuntando consistentemente varias ventajas de la ayuda entre iguales. A continuación analizaremos los resultados, de los estudios mas rigurosos, en cuatro áreas: a) en los alumnos entrenados en los sistemas de ayuda entre iguales, b) entre los usuarios del sistema de ayuda de iguales, c) el impacto de la ayuda entre iguales sobre agresores y victimas, y d) el impacto sobre el clima social y las relaciones interpersonales en la escuela.

\section{A) Resultados en los alumnos entrenados en sistemas de ayuda entre iguales}

En el estudio realizado por Cowie (1998) todos los entrevistados, tanto profesores como alumnos, coincidieron en informar que los alumnos ayudantes habían obtenido beneficios personales por su participación en el proyecto. Los beneficios más mencionados fueron un incremento en la seguridad en si mismos, y un sentimiento de responsabilidad y creencia en que estaban contribuyendo positivamente al desarrollo diario de la escuela. El 
$60 \%$ de los ayudantes informaron que estos beneficios surgieron directamente del entrenamiento recibido.

En el informe de mayor magnitud, realizado por Naylor y Cowie (1999), el 78\% de los ayudantes de iguales informaron haber ganado desde el entrenamiento útiles habilidades sociales e interpersonales, tales como escucha activa. El 58\% informo que a través de la práctica del programa habían sido capaces de demostrar que cuidaban de los compañeros necesitados y que eran capaces de desarrollar sus deseos altruistas de combatir el problema del bullying en sus escuelas.

Cowie, Taylor, Talamelli, Chauhan y Smith (2002) también encontraron que los ayudantes de iguales apreciaban la oportunidad que tenían de dirigirse hacia un problema real en sus escuelas, valorando positivamente las habilidades y los medios dados para combatir dichos problemas. Un frecuente comentario fue que la experiencia de participar en el proyecto de ayuda entre iguales les había llevado a elegir para sus estudios universitarios una profesión asistencial de ayuda y cuidado hacia los demás.

En el informe de la Fundación de Salud Mental (2002), el 93\% de los profesores manifestaron que el programa de ayuda entre iguales había mejorado las habilidades de comunicación de los estudiantes.

Por su parte, Menesini, Codecasa, Benelli y Cowie (2003) a través de una intervención basada en el sistema de compañeros amigos, destacaron que los mayores beneficios se produjeron entre los agresores, especialmente, cuando estos participaron en el entrenamiento, desarrollándose en ellos una mayor conciencia sobre los efectos de su conducta.

\section{B) Resultados entre los usuarios de la ayuda de iguales}

En este apartado, el estudio realizado por Naylor y Cowie (1999), sobre 2313 estudiantes y 234 profesores pertenecientes a 591 escuelas, halló que la ayuda entre iguales era percibida como beneficiosa por los usuarios, ya que el programa proporcionaba a alguien que les escuchaba y ayudaba a superar el problema. Además, la existencia de un sistema de ayuda entre iguales era percibido por las víctimas como indicador de que la escuela estaba actuando y se preocupaba acerca de su bienestar. De los 65 alumnos que fueron usuarios del sistema, el 82\% lo consideró útil y les dio fuerza para afrontar el bullying. El 80\% dijo que 
recomendaría el sistema a algún amigo, y solo el 18\% tuvo pensamientos negativos acerca del programa, expresando por ejemplo, que no lo encontraron útil o fue desagradable.

Smith y Watson (2004), en un estudio realizado a nivel nacional en centros de primaria y secundaria del Reino Unido, en cuya evaluación participaron 834 alumnos y 204 profesores, hallaron que el uso de los sistemas de ayuda entre iguales variaba entre las escuelas, e identificaron que a menudo dependía del papel y las horas de dedicación que asumían los ayudantes. Alrededor de la mitad de los estudiantes (53\% en primaria, y 52\% en secundaria) tenían conocimiento de alguien que había hecho uso de la ayuda entre iguales. A los estudiantes que habían usado el sistema se les preguntó el grado en que lo encontraron útil. En general, el 44\% de los usuarios en escuelas de primaria dijeron que era bastante útil, el $50 \%$ lo calificaron como algo útil, y el 6\% no lo encontró útil. En escuelas de secundaria, el $47 \%$ lo calificaron como bastante útil, el $42 \%$ dijeron que era algo útil, y el $11 \%$ restante afirmó que no era de utilidad. No hubo diferencias de genero en escuelas de primaria, pero en escuelas de secundaria más chicos que chicas dijeron que no era de ayuda (18\% vs. $7 \%$ ).

Igualmente, en un estudio más pequeño realizado por Cowie y Olafsson (2000), las victimas valoraron positivamente el tener un igual dispuesto a escuchar sus problemas, y encontraron útil la protección que les daba la presencia de ayudantes de iguales en la escuela.

En una detallada intervención realizada sobre dos clases de secundaria, Menesini et al. (2003), expresaron que las víctimas presentaban actitudes muy positivas hacia el programa de ayuda entre iguales, pero comprensiblemente, eran bastante cautelosas a la hora de acudir a un alumno ayudante que anteriormente era identificado como un agresor, y manifestaban su preferencia de ser ayudado por otro alumno del programa.

\section{C) Impacto de la ayuda entre iguales en agresores y víctimas}

En este apartado los resultados obtenidos son menos evidentes. Carecemos de evidencias cuantitativas de que la incidencia del bullying es reducida por los sistemas de ayuda entre iguales. Sin embargo, datos subjetivos sugieren que el bullying es reducido mediante estos programas. En el informe de la Fundación de Salud Mental (2002), el 61\% del personal docente percibió que la ayuda entre iguales había reducido la incidencia del bullying. 
Smith y Watson (2004) expresan que a pesar de la falta de evidencia objetiva y directa, los profesores a menudo informan de una reducción en la ocurrencia de pequeños incidentes de bullying, percibiendo a los estudiantes más capaces de resolver las situaciones por ellos mismo de forma pacífica. Muchos profesores informan sobre cómo tras la implantación del programa los estudiantes más vulnerables son detectados y ayudados más fácilmente, debido al incremento de la conciencia por parte de los estudiantes.

Por otro lado, Naylor y Cowie (1999) indicaron que los métodos de ayuda entre iguales no redujeron la incidencia del bullying, siendo los resultados similares a los de otros informes de la época (Whitney y Smith, 1993). No obstante, los usuarios concluyeron que la presencia de sistemas de ayuda entre iguales redujo el impacto negativo del bullying e hizo más aceptable para ellos informar sobre el mismo.

Cowie y Olafsson (2000) administró el cuestionario de Olweus antes de la introducción del programa de ayuda entre iguales y 7.5 meses después. Los resultados muestran que, aunque la incidencia del bullying es similar en ambas fechas, se produjo un incremento en el número de victimas que comentaban su sufrimiento a un profesor o amigo.

Al mismo tiempo, Menesini et al. (2003), encontraron resultados positivos relacionados con la prevención de conductas y actitudes negativas entre los escolares, destacando la ruptura del silencio de los testigos ante conductas violentas, y el desarrollo de sentimientos de empatía y responsabilidad entre los compañeros. Los mayores efectos se dieron en los agresores y observadores, produciéndose en ellos un mayor conocimiento de las consecuencias de sus conductas anteriores. Este efecto fue incluso mayor cuando los alumnos identificados como agresores participaron en la formación realizada para los ayudantes.

Del mismo modo, Fernández, Villaoslada y Funes (2002), informaron del éxito de un programa de mediación de iguales, a través del cual se produjo la resolución satisfactoria del $85 \%$ de los conflictos del centro. El programa resultó ser eficaz para combatir el problema de la violencia, recogiéndose un descenso en el índice de agresiones.

\section{D) Impacto de la ayuda entre iguales sobre el clima social y las relaciones interpersonales}

En el estudio realizado por Cowie (1998) el 63\% de los alumnos ayudantes creían que el servicio había tenido impacto en el conjunto de la escuela. Otros informaron que la escuela 
había llegado a ser un lugar donde era más aceptado hablar sobre dificultades emocionales y sociales. Los adultos encargados de los proyectos fueron unánimes en confirmar que el trabajo de los ayudantes fue más allá de ayudar a alumnos con dificultades y que la intervención había afectado positivamente al conjunto de la escuela.

En un estudio longitudinal Cowie et al. (2002), advirtió que al finalizar la investigación una proporción más pequeña de víctimas (8\%) usaban el sistema de ayuda entre iguales, en comparación con el estudio realizado al inicio (20\%). Ellos concluyeron que la presencia en estas escuelas de un sistema de ayuda entre iguales durante tres años o más había mejorado la atmósfera de cuidado, creando un medio donde era aceptado compartir problemas con otras personas, no siendo necesariamente un ayudante. Ellos apuntaron que las víctimas que no habían usado la ayuda entre iguales, alrededor del $40 \%$, fue porque habían recibido ayuda de otra persona, a menudo un amigo. Su interpretación fue confirmada por el porcentaje relativamente bajo de víctimas de maltrato en estas escuelas que no comunicaron a nadie su malestar, alrededor del $14 \%$, lo cual sugiere que una cultura informal de ayuda entre iguales fue fomentada por la presencia de un proyecto más formal.

En el informe de la Fundación de Salud Mental (2002), el 96\% del personal docente advirtió que el proyecto de ayuda entre iguales mejoró las relaciones y el apoyo prestado entre los jóvenes, el 100\% manifestó un incremento en la percepción de la escuela como una “comunidad que cuida", el $82 \%$ indicó que la ayuda prestada redujo los niveles de estrés en los estudiantes necesitados, y el $61 \%$ comentó que se había producido una mejora en la comunicación entre estudiantes y profesores.

\section{Conclusiones}

\section{Consideraciones prácticas}

Las investigaciones llevadas a cabo en Europa arrojan evidencia sobre que la ayuda entre iguales es un tipo de intervención efectiva contra la violencia en las escuelas (Cowie et al. 2002; Naylor y Cowie, 1999). Aunque los programas de ayuda entre iguales no siempre reducen la incidencia de la violencia, estos pueden ser una efectiva medida preventiva, y una fuente de apoyo para las víctimas. El resultado con mayor evidencia de los programas de 
ayuda entre iguales es el beneficio obtenido por los alumnos/as ayudantes. Estos beneficios se concretan en una mejora de la autoestima, una mayor seguridad en si mismos, un incremento de las habilidades sociales y de comunicación, una mayor empatía, y un sentimiento de responsabilidad y orgullo por hacer algo que contribuye a la mejora de la escuela. Estos datos están basados en autoinformes de ayudantes y en la observación de profesores, lo cual indica que puede haber elementos de deseabilidad social presentes en las respuestas. Sería útil completar estos datos con algunas medidas más objetivas de, por ejemplo, la autoestima y las habilidades sociales antes y después del entrenamiento, comparándolas quizás con las puntuaciones obtenidas por sujetos controles procedentes de la lista de espera de voluntarios del programa.

Esto no quiere decir que no existan problemas para los alumnos ayudantes. Nosotros hemos identificado 3 áreas principales de dificultades: estatus, desequilibrio de género, y ansiedad por la falta de usuarios.

En cuanto al estatus, mientras la mayoría de los ayudantes se sienten respetados y valorados, en algunos casos pueden sentir el rechazo o aislamiento de una minoría (Cowie y Olafsson, 2000; Naylor y Cowie, 1999), o pueden encontrar alguna burla sobre su figura de ayudante. Esto es más probable que suceda en escuelas donde el programa de ayuda entre iguales no está arraigado en la cultura del centro, así como en educación secundaria, donde los niños tienden a tener actitudes menos positivas hacia estos programas.

También, un buen equilibrio en el género de los ayudantes es importante. Es ampliamente informado ser más sencillo reclutar chicas que chicos para formar parte de los alumnos ayudantes. Incluso, cuando los chicos fueron reclutados su índice de abandono fue mayor, aparentemente a causa de la "presión de otros chicos". Naylor y Cowie (1999) hallaron que los chicos están perfectamente capacitados para mostrar cualidades de cuidado y ayuda, pero ellos sólo las desarrollarán cuando no se encuentre amenazada su masculinidad, por ejemplo, en colegios sólo para varones. Una explicación puede ser las representaciones de los roles de masculinidad y feminidad transmitidos por padres, iguales y medios de comunicación, asociándose habitualmente las conductas de ayuda al rol femenino. Por ello, seleccionar equitativamente el género de los alumnos ayudantes y de los profesores coordinadores de los programas, así como disponer de ayudantes varones de alto estatus que 
puedan anunciar el proyecto y llevar a cabo reuniones de información entre sus compañeros, son importantes medidas a tomar para tratar de solventar esta dificultad.

Finalmente, los ayudantes se sienten más respetados y con mayor confianza en su papel si pueden poner en práctica sus habilidades. Los peligros son, por ejemplo, que nadie acuda al servicio de ayuda por miedo a ser estigmatizados. Este bajo o escaso uso del sistema puede ser evitado, ya que los ayudantes juegan un importante papel activo en la organización de juegos y actividades (Demetriades, 1996), los cuales pueden ser atractivos para muchos niños, y a su vez, pueden permitir a estudiantes víctimas o tímidos conseguir apoyo de una forma menos obvia. Además, los programas de ayuda entre iguales pueden hacer uso de la red interna del colegio, o bien, ser usados de una forma amplia para el apoyo de iguales en diferentes áreas, tanto académicas como interpersonales, para así evitar que los alumnos que buscan los servicios del programa sean estigmatizados. (Cartwright, 2005; Cowie y Hutson, 2005; Smith y Watson, 2004).

Por otro lado, una minoría que va desde el 6\% en Smith y Watson (2002), al 18\% en Naylor y Cowie (1999), informa no encontrar útil el sistema. No disponemos de suficiente información sobre por qué algunos usuarios tienen experiencias negativas, pero estas suelen ser atribuidas a la actitud de los ayudantes, o a la falta de seguimiento. Ambas circunstancias pueden ser potencialmente solucionadas con un buen entrenamiento y supervisión del programa, así como, mediante la integración del sistema en una política antibullying que involucre a toda la escuela.

En comparación con los datos sobre los beneficios de la ayuda entre iguales en usuarios y ayudantes, donde los datos se basan en medidas de autoinformes y hacen frente a la validez, los resultados sobre el impacto en las relaciones interpersonales son más indirectos. Muchos profesores y alumnos creen que hay efectos positivos, a nivel general, sobre la incidencia del bullying en la escuela, o sobre las relaciones entre iguales, pero muchos están también inseguros sobre los resultados del programa. Por ello, se hace necesaria la obtención de datos basados en medidas más objetivas de estas variables de convivencia. Sólo dos estudios han hecho esto último, Cowie y Olafsson (2000) y Naylor y Cowie (1999), concluyéndose en ellos que el desarrollo de un programa de ayuda entre iguales, por sí solo, no parece reducir el bullying, pero sin embargo, su presencia hace percibir a estudiantes y profesores una disminución del maltrato ocurrido en la escuela. Esto ilustra la necesidad de 
más investigaciones cuantitativas y sus dificultades de interpretación, así como que resultados positivos a nivel general no pueden darse por seguros sin evidencias objetivas y cuantitativas a su favor. Serían necesarias, de forma urgente, más investigaciones para determinar en qué medida y cómo los programas de ayuda entre iguales reducen la violencia escolar. No obstante, las investigaciones de Menesini et al. (2003) y Fernández et al. (2002), en las que se trabaja directamente tanto con víctimas como con agresores, informan de una disminución de los episodios de bullying, pudiendo ser un factor determinante de estos resultados el trabajo realizado por los ayudantes con ambas partes implicadas en la violencia escolar, y no centrando su ayuda únicamente en las víctimas.

Respecto al impacto de los programas de ayuda entre iguales sobre la atmósfera general de relaciones en el centro, en un primer nivel de análisis se detecta que a la mayoría de los estudiantes no les gusta el bullying y ven en la ayuda entre iguales una buena idea para combatirlo. Pero, a un nivel de análisis mas profundo volvemos a encontrarnos con la carencia de datos objetivos sobre los efectos de la intervención en el clima social escolar, en las relaciones interpersonales, y sobre la seguridad percibida en la escuela. Como ocurre con la incidencia del bullying, generalmente obtenemos por parte de los profesores y estudiantes percepciones positivas sobre estos asuntos, pero sería necesario el uso de diseños pre-post y el empleo medidas más objetivas en la evaluación del clima social, para poder determinar con mayor exactitud el impacto de la ayuda entre iguales sobre la atmósfera del centro.

\section{Rol de los niños como participantes activos en la investigación}

Tradicionalmente, los adultos como expertos en el desarrollo infantil reúnen evidencias objetivas acerca de los niños. Más recientemente, los investigadores están viendo la necesidad de recoger las propias perspectivas de los niños, en base a que son ellos la principal fuente de información sobre cómo experimentan sus vidas. En otras palabras, hay un movimiento dirigido a investigar con niños más que sobre niños (Veale, 2005), donde los niños son participantes activos del proyecto. En este sentido, otra sugerencia es fomentar un mayor uso de los jóvenes como investigadores (Greene y Hogan, 2005). En nuestra cultura, no tenemos una fuerte tradición de escuchar a los niños, o comprender sus vidas en sus propios términos, y como principal fuente de evidencia. Sin embargo, la mayoría de los datos informados en esta revisión se fundamentan sobre las perspectivas y puntos de vista de los 
jóvenes involucrados en programas de ayuda entre iguales, tanto usuarios como ayudantes, pudiendo ser este campo un punto de partida ideal para introducir y verificar métodos de evaluación más innovadores centrados en los niños.

Por otro lado, la legislación en Europa indica que los niños deberían ser educados para ejercer como ciudadanos activos, y que las escuelas deberían proporcionar un equilibrio entre derechos, expectativas, y deberes. El derecho de los niños a participar en la toma de decisiones esta establecido en el articulo 12.1 de la Convención de las Naciones Unidas sobre los Derechos Infantiles (1989), donde se recomienda que la visión de los niños reciba su debida importancia, en función de su edad y madurez. En el año 2005, bajo la iniciativa del Consejo de Europa, jóvenes de diferentes países prepararon los Estatutos Europeos para las Escuelas Democráticas Sin Violencia. Dichos estatutos están basados en los valores y principios fundamentales compartidos por todos los europeos, y en concreto, desarrollados a partir de lo establecido en la Convención para la Protección de los Derechos Humanos y las Libertades Fundamentales del Consejo de Europa.

Este grupo de jóvenes recomendó a las escuelas de toda Europa el uso de esta declaración como modelo a partir del cual poder avanzar en el desarrollo de escuelas democráticas sin violencia. Los siete puntos de la declaración son:

1. Todos los miembros de la comunidad escolar tienen derecho a una escuela segura y pacífica, así como la responsabilidad de contribuir a crear un entorno positivo para el aprendizaje y el desarrollo personal.

2. Todos los miembros de la comunidad escolar tienen derecho a un trato de igualdad y respeto sin tener en cuenta cualquier diferencia personal, así como derecho a la libertad de expresión sin riesgos de discriminación o represión.

3. La comunidad escolar debe garantizar que todos sus miembros sean conocedores de sus obligaciones y derechos.

4. Cada escuela democrática tiene un órgano de toma de decisiones elegido democráticamente, compuesto por representantes de estudiantes, maestros, padres, y otros miembros de la comunidad escolar. Todos los miembros de este organismo tienen derecho a voto. 
5. En una escuela democrática los conflictos son resueltos de forma constructiva y no violenta en colaboración con todos los miembros de la comunidad escolar. Cada escuela tiene personal docente y estudiantes entrenados en la prevención y solución de conflictos, a través del asesoramiento y la mediación.

6. Todos los casos de violencia son investigados y tratados con prontitud, independientemente de si los involucrados son estudiantes u otros miembros de la escuela.

7. La escuela forma parte de una comunidad local. Por tanto, la colaboración e intercambio de información con los agentes locales es esencial para la prevención y solución de problemas.

En este sentido, los programas de ayuda entre iguales impulsan y facilitan la participación de los niños y jóvenes en las decisiones que afectan a sus vidas, y les ayudan a resolver los problemas de violencia que muchos de ellos observan o sufren diariamente en sus escuelas. 


\section{Referencias}

Batson, C. D., Ahmad, N., Lishner, D. A. y Tsang, J-A. (2002). Empathy and altruism. En C. R. Snyder y S. L. Lopez (eds.), Handbook of positive psychology (pp. 485-498). Oxford: Oxford University Press.

Borzekowski, D.L.G. y Rickert, V.I. (2001). Adolescents, the Internet, and health: issues of access and content. Journal of Applied Developmental Psychology, 22 (1), 49-59.

Boulton, M. J., Trueman, M., Chau, C., Whitehand, C. y Amatya, K. (1999). Concurrent and longitudinal links between friendship and peer victimisation: implications for befriending interventions. Journal of Adolescence, 22, 461-466.

Cartwright, N. (2005). Setting up and sustaining peer support systems in a range of schools over 20 years. Pastoral Care in Education, 23 (2), 45-50.

Cowie, H. (1998). Perspective of teachers and pupils on the experience of peer support against bullying. Educational Research and Evaluation, 4, 108-125.

Cowie, H. y Hutson, N. (2005). Peer Support: a strategy to help bystanders challenge school bullying. Pastoral Care in Education, 23 (2), 40-44.

Cowie, H. y Olafsson, R. (2000). The role of peer support in helping the victims of bullying in a school with high levels of aggression. School Psychology Internacional, 21, 79-95.

Cowie, H. y Wallace, P. (2000). Peer Support in Action. London: Sage.

Cowie, H., Boardman, C., Dawkins, J. y Jennifer, D. (2004). Emotional Health and Wellbeing: a Practical Guide for Schools. London: Sage.

Cowie, H., Naylor, P., Talamelli, L., Chauhan, P. y Smith, P. K. (2002). Knowledge, use of and attitudes towards peer support. Journal of Adolescence, 25, 453-467.

Cresser, F., Gunn, L. y Balme, H. (2001). Women's experiences of on-line e-zine publication. Media culture and society, 23 (4), 457-473.

Fernandez, I., Villaoslada, E. y Funes, S. (2002). Conflicto en el centro escolar. Madrid: Catarata.

Greene, S. y Hogan, S. (Eds.). (2005). Researching children's experience. London: Sage.

McKenna, K. Y. A. y Bargh, J. A. (2000). Plan 9 from cyberspace: The implications of the Internet for personality and social psychology. Personality and Social Psychology Review, 4 (1), 57-75.

Menesini, E., Codecasa, E., Benelli, B. y Cowie, H. (2003). Enhancing children's responsibility to take action against bullying: evaluation of a befriending intervention in Italian middle schools. Aggressive Behavior, 29, 1-14. 
Mental Health Foundation (2002). Peer support: Someone to turn to. An evaluation report of the Mental Health Foundation Peer Support Programme. London y Glasgow: Mental Health Foundation.

Naylor, P. y Cowie, H. (1999). The effectiveness of peer support systems in challenging school bullying: the perspectives and experiences of teachers and pupils. Journal of Adolescence, 22, 467-479.

Salmivalli, C., Lagerspetz, K., Björkqvist, K., Österman, K. y Kaukiainen, A. (1996). Bullying as a group process: participant roles and their relations to social status within the group. Aggressive Behavior, 22, 1-5.

Schulman, M. (2002). How we become moral. En C. R. Snyder y S. L. Lopez (Eds.), Handbook of Positive Psychology, (pp. 499-512). Oxford: Oxford University Press.

Seligman, M. E. P., Reivich, K., Jaycox, L. y Gillham, J. (1995). The Optimistic Child. New York: Houghton Mifflin.

Smith, P. K. y Watson, D. (2004). Evaluation of the CHIPS (ChildLine in Partnership with Schools) programme. Research report RR570. Nottingham: DfES publications.

United Nations (1991). United Nations Convention on the Rights of the Child. Innocenti Studies. Florence: UNICEF.

Veale, A. (2005). Creative methodologies in participatory research with children. En S. Greene y D. Hogan (Eds.), Researching Children's Experience (pp. 253-272). London: Sage.

Whitney, I. y Smith, P. K. (1993). A survey of the nature and extent of bully/victim problems in junior/middle and secondary schools, Educational Research, 35, 3-25.

Wolak, J., Mitchell, K. J., y Finkelhor, D. (2002). Close online relationships in a national sample of adolescents. Adolescence, 37 (3), 441-455.

Ybarra, M. L. 2004. Linkages between depressive symptomatology and Internet harassment among young regular Internet user. CyberPsychology and Behaviour, 7 (2), 248-257.

Ybarra, M. L. y Mitchell, K. J. (2004). Online aggressors, victims and aggressors/victims: a comparison of associated youth characteristics. Journal of Child Psychology and Psychiatry, 45 (7), 1308-1316. 